\title{
Verfahrensanweisung für die lod-131 Ganzkörperszintigrafie beim differenzierten Schilddrüsenkarzinom (Version 5)
}

Frederik A. Verburg ${ }^{1,2}$, Matthias Schmidt ${ }^{1,3}$, Michael C. Kreiss| ${ }^{1,4}$, Frank Grünwald ${ }^{1,5}$, Michael Lassmann ${ }^{1,6}$, Heribert Hänscheid ${ }^{1,6}$, Melanie Hohberg1,3, Markus Luster $^{1,7}$, Markus Dietlein ${ }^{1,3}$

1 für die Deutsche Gesellschaft für Nuklearmedizin (DGN)

2 Klinik für Nuklearmedizin des Universitätsklinikums Marburg

3 Klinik und Poliklinik für Nuklearmedizin, Uniklinik Köln

4 Klinik für Radiologie und Nuklearmedizin, Universitätsklinikum Magdeburg

5 Klinik und Poliklinik für Nuklearmedizin der Universität Frankfurt

6 Klinik und Poliklinik für Nuklearmedizin der Universität Würzburg

7 Universitätsklinikum Gießen und Marburg, Standort Marburg, Klinik für Nuklearmedizin

\section{ZUSAMMENFASSUNG}

Die Version 5 der Verfahrensanweisung für die lod-131 Ganzkörperszintigrafie beim differenzierten Schilddrüsenkarzinom ist ein Update der Version 4, publiziert durch die Deutsche Gesellschaft für Nuklearmedizin (DGN). Diese Verfahrensanweisung beschreibt mit primärer Zielsetzung die fachgerechte Durchführung der Ganzkörperszintigrafie nach Applikation einer diagnostischen bzw. einer therapeutischen I-131 Aktivität. Die Verfahrensanweisung wurde von einer repräsentativen Expertengruppe im Konsensverfahren verabschiedet. Sie entspricht damit einer Verfahrensanweisung der ersten Stufe (S1) nach den Kriterien der Arbeitsgemeinschaft der Wissenschaftlichen Medizinischen Fachgesellschaften (AWMF).

\section{Zielsetzung}

Ziel dieser Leitlinie ist die Unterstützung nuklearmedizinisch tätiger Ärzte bei der Indikationsstellung und Durchführung der I-131 (Radioiod) Ganzkörperszintigrafie (GKS) zum Nachweis oder Ausschluss von postoperativ verbliebenem Restschilddrüsengewebe, iodspeichernden Rezidiven und/oder iodspeichernden Metastasen bei papillär und follikulär differenzierten Schilddrüsenkarzinomen und ihren Varianten sowie beim wenig differenzierten Schilddrüsenkarzinom. Dabei steht GKS als Oberbegriff für eine oder mehrere szintigrafische Aufnahmen des gesamten Körpers, statische Aufnahmen von Teilbereichen, z. B. Hals-Thorax mit längerer Messdauer oder von SPECT-Aufnahmen mit oder ohne CT zur morphologischen Korrelation und Bildkorrektur. Zu Einsatz und Durchführung anderer diagnostischer Verfahren in der Behandlung des Schilddrüsenkarzinoms wird auf die entsprechenden nationalen und internationalen Leitlinien verwiesen [15, 32, 62]. Der im Rahmen dieser Verfahrensanweisung eingesetzte Terminus „initiale Radioiodtherapie“ steht für die erste therapeutische Anwendung von Radioiod bei einem Patienten. Diese dient:

1. der Beseitigung von Schilddrüsenrestgewebe im Sinne einer ablativen Radioiodtherapie

2. der adjuvanten Behandlung zur Therapie eventuell vorhandener okkulter Metastasen

3. dem hochspezifischen und -sensitiven Staging mittels posttherapeutischer GKS

4. bei bereits bekannter Metastasierung bzw. bei bekanntem Resttumor: der (ersten) Behandlung der Manifestationen

\section{Hintergrundinformation}

Ein (benigner) Schilddrüsenrest oder ein differenziertes, von den Thyreozyten ausgehendes Karzinomgewebe ist dann szintigrafisch nachweisbar, wenn das aufgenommene radioaktive lod eine erkennbar erhöhte Zählrate über dem lokalen Untergrund bewirkt [13]. Die Sensitivität der Bildgebung verbessert sich bei höherer Zählstatistik, durch verringertes Bildrauschen und verschlechtert sich bei erhöhtem Untergrund. Mögliche Strategien zur Steigerung der Detektionsrate sind die Verabreichung einer höheren I-131 Aktivität, eine Verlängerung der Messzeit, ein leistungsstarkes Detektorsystem und ein optimierter Zeitabstand zwischen der Radioiodgabe und der Datenakquisi-
ABKÜRZUNGEN
RIT Radioiodtherapie
GKS Ganzkörperszintigrafie
rxGKS Ganzkörperszintigrafie nach Radioiod- therapie
dxGKS Ganzkörperszintigrafie nach diagnosti- scher Aktivität
TSH Thyreoidea Stimulierendes Hormon
rhTSH rekombinantes humanes TSH
Tg Thyreoglobulin
SPECT/CT Single Photon Emission Computed Emission Tomography/Computed Tomography 
tion. Die auf eine Radioiodtherapie (RIT) folgende Ganzkörperszintigrafie (rxGKS) besitzt aufgrund der deutlich höheren eingesetzten I-131 Aktivität auch eine höhere Sensitivität als die Ganzkörperszintigrafie nach Verabreichung einer diagnostischen Aktivität (dxGKS) [30, 56].

Die Erkennbarkeit des lod-speichernden Gewebes in der Bildgebung hängt zudem von seiner Masse sowie von der spezifischen Fähigkeit zur lodaufnahme und lodspeicherung ab. Eine Expression des Natrium-Iodid-Symporters (NIS) kann insbesondere bei den gering differenzierten Schilddrüsenkarzinomen und bei den aggressiven Varianten der papillären und follikulären Schilddrüsenkarzinome fehlen oder nur unzureichend vorhanden sein [5]. Des Weiteren kann die Fähigkeit zur lodaufnahme bei hochdifferenzierten Karzinomen im Verlauf der Erkrankung oder nach vorangegangenen Radioiodtherapien abnehmen.

Die Sensitivität der dxGKS zur Detektion regionaler oder distanter Metastasen hängt sehr von dem untersuchten Patientenkollektiv und den eingesetzten Methoden (ausschließlich planare Aufnahmen vs. SPECT vs. SPECT/CT) ab. In der Literatur finden sich hier Werte zwischen 60-90\% [7, $14,37,77,78,90,91]$, wobei sich insbesondere der Einsatz der Hybridbildgebung mittels SPECT/CT als vorteilhaft erwiesen hat. Die gewebsspezifische Aufnahme von I-131 führt zu einer sehr hohen Spezifität und einem hohen positiven prädiktiven Wert der Untersuchung. Da der Nachweis einer lodaufnahme in Metastasen auch für die Therapierbarkeit des Tumors mit I-131 entscheidend ist und die Indikation zur RIT wesentlich mitbestimmt, besitzt diese funktionelle Bildgebung auch deshalb einen hohen klinischen Stellenwert.

\section{Indikationen}

Nach den rechtlichen Vorgaben in Deutschland (Strahlenschutzgesetz, Richtlinie Strahlenschutz in der Medizin) ist die individuelle Indikation zur Verabreichung von I-131 durch einen Arzt mit entsprechender Fachkunde zu stellen [80, 81]. Inhaltlich sind hierbei die aktuell gültigen Leitlinien sowie die hierauf basierenden regionalen und überregionalen „standard operating procedures“ (SOPs, z. B. im Netzwerk onkologischer Spitzenzentren) zu berücksichtigen [57]. Als Kriterien für die individuelle Indikationsstellung eignen sich das initiale Risikoprofil des Patienten, das Resektionsausmaß, vorangegangene Therapien, die Ergebnisse früherer Szintigrafien und anderer bildgebender Verfahren, der Thyreoglobulin (Tg)-Spiegel unter endogener oder exogener TSH-Stimulation bzw. unter Suppression bei Verwendung eines sensitiven Thyreoglobulin-Assays (funktionelle Assay-Sensitivität von zumindest 0,1 bis $0,3 \mathrm{ng} / \mathrm{ml}$ ) sowie der Nachweis oder das Fehlen von Anti-Tg-Antikörpern durch eine direkte Bestimmung der Antikörper. Ergänzend kann eine Tg-Wiederfindung zum Ausschluss seltener Störquellen, wie z. B. heterophiler An- tikörper, erfolgen. Bei der Bewertung des initialen Risikoprofils sind neben den internationalen Leitlinien und den Ergebnissen prospektiver Studien auch die aktuell 8. Auflage der TNM-Klassifikation sowie die WHO-Klassifikation der Tumorentitäten in der aktuell 4. Auflage zu berücksichtigen $[43,48,65,89]$.

Mit einem ausreichenden zeitlichen Abstand zur initialen RIT - bei unkompliziertem Verlauf etwa 6-12 Monate - erfolgt anhand laborchemischer, sonografischer und szintigrafischer Verfahren eine Neubewertung des individuellen Risikos [88, 92, 95].

Abhängig vom Zeitpunkt innerhalb der Behandlungskette und vom Verlauf der Erkrankung können folgende Indikationen zur l-131 Szintigrafie bestehen:

A. Diagnostik bzw. Dosimetrie vor RIT

B. Bildgebung wenige Tage nach RIT (I-131 rxGKS)

C. Erfolgskontrolle und Re-Staging 6 - 12 Monate nach der initialen Radioiodtherapie (I-131 dxGKS)

D. Diagnostik (I-131 dxGKS) nach individueller Indikationsstellung bei

D.1. Patienten ohne Therapieerfolg nach initialer Radioiodtherapie

D.2. Patienten mit erhöhten Anti-Thyreoglobulin-Antikörpern oder bei gestörter Tg-Wiederfindung

D.3. Patienten mit persistierender Erkrankung

D.4. Patienten mit Rezidiv(verdacht)

\section{Diagnostik bzw. Dosimetrie vor RIT}

Vor einer RIT ist die Verwendung höherer diagnostischer I-131 Aktivitäten ungünstig, da dies durch Vorschädigung gut speichernden Zielgewebes dessen lodkinetik nachweislich beeinträchtigt, die unter Therapie erreichte Dosis reduziert [46] und somit die Therapie-Erfolgsrate verringern kann [96]. Dieser Effekt wird mit dem Begriff „Stunning“ beschrieben. Da es Hinweise darauf gibt, dass Stunning innerhalb der ersten Tage nach diagnostischen Aktivitäten noch nicht oder weniger stark ausgeprägt ist [99], sollte eine RIT, deren Indikation sich erst aus einer dxGKS in hypothyreoter Stoffwechsellage ergibt, so früh wie möglich innerhalb von wenigen Tagen nach der dx GKS erfolgen. Sollte eine RIT von distanten Metastasen unter einer endogenen TSH-Stimulation nicht möglich sein, wie z. B. nach Stimulation mittels rekombinantem humanem TSH (rhTSH), so empfiehlt sich ein Zeitabstand von etwa 3 Monaten zwischen dxGKS und nachfolgender RIT. 
FRAGE 1

Ist eine I-131 Szintigrafie vor der initialen Radioiodtherapie nötig?

Kommentar: Eine I-131 Szintigrafie kann vor allem dann vor der initialen RIT sinnvoll sein, wenn das Ergebnis die Indikation zur RIT oder die Aktivität der RIT verändern könnte. Falls I-131 vor der initialen RIT eingesetzt wird, sind niedrige Aktivitäten bis $10 \mathrm{MBq}$ I-131 zu empfehlen. Unter Berücksichtigung eines möglichen Stunning durch eine I-131 Szintigrafie vor einer RIT sollte die Verwendung von Tc-99m Pertechnetat (= lodäquivalent), I-123 oder I-124 als Alternativen geprüft werden [6, 24].

Eine geringe Aktivität von unter $10 \mathrm{MBq}$ l-131 kann vor der ersten RIT zur prätherapeutischen, quantitativen Szintigrafie der Halsregion eingesetzt werden, wenn die Größe des Schilddrüsenrests durch den Operationsbericht, durch die Sonografie oder durch andere funktionell-bildgebende Verfahren (Tc-99m Pertechnetat, I-123, I-124) nur unzureichend abzuschätzen ist, oder wenn das Ergebnis der quantitativen Szintigrafie die Entscheidung zur RIT oder die Höhe der I-131-Therapieaktivität (z. B. bei Hoch-Risiko-Konstellation) beeinflusst [15]. Bei Hoch-Risiko-Patienten, insbesondere bei (Verdacht auf) Metastasierung kann vor einer geplanten RIT eine Ganzkörperszintigrafie mit 10 MBq I-131 zur Dosimetrie oder zur Detektion von iodavidem Gewebe mit Speicherungen von $\geq 0,1 \%$ sinnvoll sein [45].

\section{Bildgebung während der RIT (I-131 rxGKS)}

Grundsätzlich ist nach jeder therapeutischen Anwendung von I-131 die rxGKS durchzuführen (Verfahrensanweisung zur Radioiodtherapie beim differenzierten Schilddrüsenkarzinom, Version 4) [15]. Die Sensitivität der Szintigrafie nach Gabe der Therapieaktivität ist höher als die Detektionsrate nach Applikation von I-131 mit Aktivitäten im diagnostischen Bereich [30, 56, 64]. Die rxGKS liefert häufig zusätzliche relevante diagnostische Informationen, z. B. die Darstellung bisher unbekannter iodavider Metastasen. Eine besondere Rolle spielt die Bildgebung im Rahmen der initialen RIT, da hier die Ultraschalldiagnostik bedingt durch postoperative Veränderungen nur eine eingeschränkte Aussagekraft hat. In einem signifikanten Anteil der Patienten werden auch in einem initialen Niedrig-Risiko-Stadium Lymphknotenmetastasen nachgewiesen, mit einer entsprechenden Änderung der TNM-Klassifikation und einer Änderung der weiteren Vorgehensweise bzw. Intensivierung der Nachsorge $[4,72,85]$.

\section{Erfolgskontrolle und Re-Staging 6-12 Monate nach der initialen RIT (I-131 dxGKS)}

Die in der „Richtlinie Strahlenschutz in der Medizin“ [80] vorgeschriebene Erfolgskontrolle der nuklearmedizinischen Behandlung sollte nach einer RIT durch eine Thy-
reoglobulin-Messung unter endogener oder exogener TSH-Stimulation in der Regel kombiniert mit einer I-131 Ganzkörperszintigrafie stattfinden. Während früher der Umfang der Nachsorge vor allem am initialen Tumorstadium ausgerichtet wurde, orientiert man sich inzwischen vor allem am Ergebnis der Response-Evaluation 6-12 Monate nach der RIT. Dabei geht es unter klinischen Gesichtspunkten v. a. um den Ausschluss iodavider Metastasen [90, 91].

Die Radioioddiagnostik sollte mit einem ausreichenden zeitlichen Abstand zur initialen RIT durchgeführt werden, da die Elimination von Restschilddrüsengewebe Zeit benötigt. Zeitabstände von $\leq 3$ Monaten haben sich als zu kurz erwiesen, da Restschilddrüsengewebe bei einer frühzeitigen Evaluation häufig zu messbaren Tg-Spiegeln und zur szintigrafisch persistierenden lodspeicherung geführt hat. Eine Diagnostik $\leq 3$ Monaten birgt damit das Risiko einer „falsch-positiven“ Befundinterpretation [98]. Bei der risikoadaptierten Verwendung niedriger l-131 Aktivitäten bei der initialen RIT (z. B. 2 GBq I-131 oder weniger) hat sich ein Zeitraum von etwa 8-12 Monaten bis zur Beurteilung des Ablationserfolges als praktikabel erwiesen [52, 71].

\section{FRAGE 2 \\ Mit welchem zeitlichen Abstand nach der initialen RIT sollte die Erfolgskontrolle durch- geführt werden? \\ Kommentar: Bei unkompliziertem Krankheitsverlauf sollte die Radioioddiagnostik (dxGKS) 6-12 Monate nach einer Therapie mit 3,7 GBq I-131 erfolgen. Nach einer Gabe von geringeren Aktivitäten (z. B. 2 GBq I-131 oder weniger) ist ein längeres Intervall von 8 Monaten bis zu 12 Monaten günstig (98).}

Sofern die Dokumentation eines Therapieansprechens erwartet wird, ist für eine durchgehend unbeeinträchtigte Lebensqualität, zur Reduzierung einer Komorbidität und zur Vermeidung einer längeren Arbeitsunfähigkeit die exogene TSH-Stimulation mit rekombinantem humanem (rh) TSH das bevorzugte Verfahren. Bei einer TSH-Stimulation mit dem rekombinanten humanen TSH ist es allerdings technisch nicht möglich, den maximalen stimulierten $\mathrm{Tg}$ Wert vor Gabe der I-131-Kapsel zu bestimmen. Deshalb ist es ein pragmatisches Vorgehen, die zweimalige Injektion von rhTSH sowohl für die stimulierte Tg-Messung als auch für die I-131 dxGKS zu nutzen. Bei Patienten ohne Hinweis auf pathologische Radioiod-Speicherherde außerhalb der Halsregion im rxGKS ist der Nachweis iodavider Fernmetastasen durch eine dxGKS mit diagnostischen Aktivitäten wenig wahrscheinlich. Bei hoher Restspeicherung im Schilddrüsenrest bei der initialen RIT ist eine szintigrafische Kontrolle des Ablationserfolgs zur Neubewertung des Risikos (unvollständiger Ablationserfolg, Verbleib iodavider Lymphknotenmetastasen) sinnvoll, da dies die weitere Behandlung und Nachsorge beeinflussen kann. Eine 
SPECT/CT erhöht die Spezifität durch die anatomische Zuordnung I-131-positiver Befunde (rechtfertigende Indikation zur SPECT/CT).

Zur Frage, wann eine initiale Therapie als erfolgreich zu bewerten ist, herrscht keine einheitliche Expertenmeinung. Insbesondere fehlen verlässliche Daten, um quantitative Grenzwerte für eine residuelle lodspeicherung im Restschilddrüsengewebe angeben zu können, die für eine Prognose relevant sind. Auch der in prospektiven Studien verwendete Grenzwert von 0,1\% der verabreichten Aktivität war letztendlich arbiträr gewählt und nie Gegenstand einer prognostischen Validierung. Da selbst Schilddrüsenreste mit einem Uptake $\leq 0,01 \%$ noch szintigrafisch nachweisbar sind [13], wird in die Wahl der weiteren Behandlungsstrategie der Tg-Spiegel integriert. In internationalen Leitlinien und Handlungsempfehlungen wird eine Restspeicherung von $\leq 0,1 \%$ in der Schilddrüsenloge üblicherweise als tolerabel angesehen [32, 51, 60], wobei dieser relativ hohe Prozentwert zu Raten erfolgreicher Ablation von $90 \%$ oder mehr führt. Hingegen werden vollständig negative $d x G K S$, was einer deutlich geringeren Restspeicherung von $\leq 0,01 \%$ entspricht [13], nur bei etwa $60 \%$ der Patienten nach initialer RIT erreicht. Bei negativer dxGKS wurden verringerte Rezidiv- und Mortalitätsraten beschrieben [92, 95]. Bei Patienten hingegen, die nach der initialen RIT nicht in vollständiger Remission sind, ist das Rezidiv- und Mortalitätsrisiko langfristig höher als bei Patienten mit erfolgreicher Initialtherapie [86]. Es ist anhand des initialen Risikoprofils und anhand einer Neubewertung des individuellen Risikos (stimuliertes Tg, Radioioddiagnostik, andere Bildgebung) für den Einzelfall zu entscheiden, ob eine erneute RIT indiziert ist.

\section{FRAGE 3}

Nach welchen Kriterien wird die initiale RIT als erfolgreich bewertet und welche Konsequenzen ergeben sich aus dieser Bewertung?

Kommentar: Verbleibt mehr als 6 Monate nach einer initialen RIT im Restschilddrüsengewebe eine I-131 Speicherung, wird in Zusammenschau mit dem stimulierten Tg-Spiegel und dem initialen Risikoprofil über Therapiekonsequenzen entschieden. Liegt der stimulierte $\mathrm{Tg}$-Wert unter $1 \mathrm{ng} / \mathrm{ml}$, erfolgt in der Regel keine weitere RIT. Die Charakteristik des verwendeten Tg-Assays ist bei der Wahl einer geeigneten Tg-Entscheidungsgrenze zu berücksichtigen. Hatte zudem ein günstiges Risikoprofil vorgelegen, ist bei niedrigem stimuliertem $\mathrm{Tg}$-Spiegel keine TSH-suppressive Einstellung anzustreben. Bei Tg-Werten $\geq 1 \mathrm{ng} / \mathrm{ml}$ sollte individuell entschieden werden. Eine zweite RIT kann insbesondere bei initial ungünstigem Risikoprofil erwogen werden. Für die Abschätzung des individuellen Risikos ist der serielle Verlauf der Tg-Werte besser geeignet als ein isolierter Tg-Messwert. Daher ist auch bei stimulierten $\mathrm{Tg}$-Werten zwischen 1 und $10 \mathrm{ng} / \mathrm{ml}$ die zweite RIT keine Standardprozedur, sondern bedarf einer individuell begründeten Indikationsstellung. Falls sich bildgebend ein metastasentypischer I-131-Speicherherd zeigt, besteht ein Behandlungsbedarf unabhängig von der Höhe des stimulierten Tg-Spiegels. In der Kombination aus I-131-Speicherherden mit niedrigen Tg-Spiegeln unter einer TSH-Stimulation sind Differentialdiagnosen einer I-131-Speicherung zu bedenken (Fallstricke der Diagnostik).

Auf eine dxGKS zur Erfolgskontrolle nach einer initialen RIT kann im Einzelfall verzichtet werden, wenn folgende Voraussetzungen erfüllt sind [1, 3, 53, 58, 67, 70, 87]:

- ein initial günstiges Risikoprofil wie TNM-Klassifikation pT1-2 p/cN0 M0 R0 ohne extrathyreoidale Ausbreitung [100]

- Tg-Spiegel bei der initialen RIT niedrig (z. B. <1 ng/ml)

- Tg-Spiegel unter Levothyroxin unterhalb der funktionellen Assaysensitivität

- Tg-Messwerte sind valide (Anti-Tg-Antikörper nicht erhöht, Tg-Wiederfindung nicht gestört), keine Hinweise auf den Einfluss heterophiler Antikörper

- die rxGKS nach der initialen RIT, wenn verfügbar inkl. SPECT(/CT), zeigte keine verdächtigen lod-Speicherherde außerhalb der Schilddrüsenloge. Hier ist zu bedenken, dass bei initial kräftiger Speicherung in einem Schilddrüsenrest in Relation dazu schwächer speichernde iodavide Metastasen bei der rxGKS maskiert sein können und sich erst bei der folgenden dxGKS nachweisen lassen.

Die in der „Richtlinie Strahlenschutz in der Medizin “ geforderte Erfolgskontrolle basiert dann auf den regelmäßigen $\mathrm{Tg}$-Kontrollen und der Sonografie. Bei auffälligen Befunden erfolgt die gezielte Indikationsstellung zur I-131 dxGKS.

\section{Diagnostik (I-131 dxGKS) nach individueller Indikationsstellung}

Liegt bei der Erfolgskontrolle $\geq 6-12$ Monate nach der I-131 Ablation der Tg-Spiegel bei der Abwesenheit von interferierenden Anti-Tg-Antikörpern nach Stimulation im Zielbereich und zeigt die dxGKS die (weitgehende) Ablation der Restschilddrüse und keine Metastasen, so ist - unabhängig vom initialen Risikoprofil - von einem individuell niedrigen Rezidivrisiko auszugehen [95]; in dieser Situation ist eine Wiederholung der dxGKS ohne weitere Hinweise auf ein Rezidiv weder bei Patienten mit initial als niedrig, noch bei Patienten mit initial als hoch eingeschätztem Risiko [10] regelhaft indiziert. 
Patienten ohne Therapieerfolg nach initialer Radioiodtherapie

Der prognostische Wert einer persistierenden lodspeicherung im Hals wird kontrovers diskutiert. Verbliebene Schilddrüsenkarzinomzellen oder normale Thyreozyten, die bei entsprechender Prädisposition später ebenfalls maligne entarten, können das Rezidivrisiko erhöhen [73]. Bei höheren Restspeicherungen sind sowohl der dxGKS als auch der Laborwert Tg nur eingeschränkt für die Diagnose eines Lokalrezidivs verwertbar. Ist bei einem $\geq 6-12$ Monate nach der initialen RIT messbaren Tg-Spiegel $\geq 1 \mathrm{ng} /$ $\mathrm{ml}$ unter einer TSH-Suppression bereits eine erneute RIT indiziert, sollte diese, um die lodkinetik im Zielgewebe nicht negativ zu beeinflussen, ohne vorherige I-131 dxGKS durchgeführt werden. Dies gilt auch für Patienten ohne eine pathologische extrathyreoidale Radioiod-Speicherung bei der primären RIT, bei denen initial ein (größerer) Schilddrüsenrest vorgelegen hatte.

Bei Patienten mit bekannten iodaviden Metastasen ist vor der Durchführung der dxGKS zu prüfen, ob bei der Diagnostik die zusätzliche Erhebung dosimetrischer Daten sinnvoll ist, wodurch ggf. im Intervall eine RIT mit individuell ermittelter I-131 Aktivität ermöglicht wird [42, 45, 93]. Der Nachweis oder dringende Verdacht auf verbliebenes Tumorgewebe (Tumorrest, Lokalrezidiv, Metastasen) begründet in aller Regel einen Behandlungsbedarf. Optionen sind eine weitere RIT, eine operative Behandlung oder eine perkutane Strahlentherapie bzw. eine Kombination aus diesen Behandlungen. Besteht anhand des Tg-Verlaufs und der Bildgebung eine nur geringe Wahrscheinlichkeit für die Persistenz der Metastasen, sollte auf die zeitnahe Durchführung einer erneuten dxGKS verzichtet werden.

Nach therapeutischen Maßnahmen (z. B. erneute OP oder weitere RIT) bei verbliebenen Tumormanifestationen nach initialer RIT kann eine I-131 dxGKS zur Erfolgskontrolle dieser Therapien indiziert sein.

Patienten mit erhöhten Anti-ThyreoglobulinAntikörpern oder bei gestörter Tg-Wiederfindung

Bei Nachweis von Anti-Tg-Antikörpern und/oder bei gestörter Tg-Wiederfindung ist ein individuelles Vorgehen erforderlich. Da bei den modernen immunometrischen Assays Anti-Tg-Antikörper nahezu ausschließlich zu erniedrigten $\mathrm{Tg}$-Werten führen, ist zwar bei erhöhtem Tg unabhängig von einer gestörten Wiederfindung und/oder erhöhten Anti-Tg-Antikörpern von einer Tumorpersistenz auszugehen, ein Tg-Spiegel im Zielbereich kann aber nicht als Beweis einer kompletten Remission betrachtet werden [79, 94]. Bei den betreffenden Patienten ist die Entwicklung des Anti-Tg-Antikörperspiegels im zeitlichen Verlauf zu berücksichtigen. Bei konstantem oder ansteigendem Spiegel ist die Wiederholung der dxGKS ggf. auch mehrfach indiziert, wobei die Dauer des Intervalls individuell bemessen wird und sich u. a. am initialen Tumorstadium, am Ergebnis vorangegangener Szintigrafien und am Verlauf der Anti-Thyreoglobulin-Antikörperspiegel (fallend bzw. konstant vs. ansteigend) orientieren sollte [94]. Bei HochRisiko-Patienten und bei Rezidivverdacht kommen neben der Sonografie der Halsweichteile und der I-131 dxGKS weitere Methoden zum Einsatz: z. B. Computertomographie (CT) des Thorax, Magnetresonanztomographie (MRT) von Hals und Mediastinum, F-18 FDG-PET/CT oder die F-18 FDG PET/MRT u.a.m. [66, 94].

Bei im Verlauf deutlich rückläufigem (d. h. $<50 \%$ vom Ausgangswert) oder nicht mehr nachweisbarem Anti-Tg-Antikörperspiegel ist bei einem Tg-Spiegel im Zielbereich von einer Remission der Erkrankung auszugehen [8, 9, 40, 41, 59]. Analog zu den Patienten mit valider Tg-Messung sollte im Fall einer bereits früher unauffälligen I-131 dxGKS auf die Wiederholung dieser Untersuchung verzichtet werden.

Patienten mit persistierender Erkrankung

Grundsätzlich sollten bei Patienten mit persistierender Erkrankung vor der Planung einer dxGKS die Konsequenzen für das weitere therapeutische Vorgehen bedacht werden. Bei optional folgender RIT von iodaviden Metastasen kann die zusätzliche Erhebung dosimetrischer Daten sinnvoll sein [12]. Die Ermittlung der maximal verabreichbaren I-131-Aktivität bei gleichzeitiger Schonung der Risikoorgane (z. B. Knochenmark) sollte sich an den Empfehlungen der entsprechenden Leitlinie der European Association of Nuclear Medicine (EANM) orientieren [45]. Ein ansteigender Tg-Wert zeigt ein Rezidiv an, so dass eine oder mehrere RIT ohne Dosimetrie indiziert sein können, insbesondere wenn keine Hinweise auf eine fehlende lodspeicherung vorliegen. Eine RIT kann ohne vorangehende I-131 dxGKS durchgeführt werden. Bei einer individuellen Indikation zur I-131 dxGKS ist auch der zeitliche Aspekt zu berücksichtigen, dass eine $d x G K S$ das Speicherverhalten einer nachfolgenden RIT negativ beeinflussen kann.

Sollte aufgrund eines histologisch geringen Differenzierungsgrads, des Vorliegens einer aggressiven Variante des differenzierten Schilddrüsenkarzinoms oder einer nur geringen I-131-Speicherung in der vorherigen I-131 rxGKS die Indikation zu einer weiteren RIT zweifelhaft sein, kann eine I-131 dxGKS zur Evaluation der Radioiod-Speicherung bekannter Metastasen, zur Metastasensuche im Rahmen des Re-Stagings oder zur Klärung einer eventuellen Operabilität, ggf. unter intraoperativen Nutzung einer Gamma-Sonde [69], sinnvoll sein. Vor allem bei Hoch-Risiko-Patienten und Patienten mit einer kurzen Tg-Verdopplungszeit $[27,39]$ ist die Diagnostik um eine F-18 FDG-PET/CT oder F-18 FDG PET/MRT zu ergänzen. Die PET veränderte bei $43 \%$ dieser Patienten die weitere Versorgung, wobei häufig I-131-negative Metastasen entdeckt wurden und in $20 \%$ der Patienten eine potentiell kurative Operation indiziert wurde [66].

Nach therapeutischen Maßnahmen (z. B. erneute OP oder weitere RIT) aufgrund einer persistierenden Erkrankung 
kann bei vorher I-131-positiven Metastasen eine erneute I-131 dxGKS zur Erfolgskontrolle dieser Therapien indiziert sein.

\section{I-131 Patienten mit Rezidiv(verdacht)}

Bei Patienten, bei denen nach vollständiger Remission ein Rezidiv histopathologisch gesichert wird oder bei denen sich laborchemisch oder bildgebend ein dringender Verdacht auf ein Rezidiv ergibt, sollte die Möglichkeit einer operativen Behandlung geprüft werden. Die Erfolgsaussichten einer diagnostischen und/oder therapeutischen Anwendung von l-131 können anhand der Ergebnisse der früheren I-131 Szintigrafien prognostiziert werden. Eine Dosimetrie oder eine qualitative Abschätzung bezüglich einer therapeutisch nutzbaren lodspeicherung kann mit I-123, I-124 oder I-131 erfolgen. Um der Möglichkeit eines Stunning zu begegnen, kann unmittelbar nach der Radioioddiagnostik eine RIT mit anschließender I-131 rxGKS durchgeführt werden. Die rxGKS ist hinsichtlich der weiteren therapeutischen Konsequenzen zu bewerten.

Der laborchemische Nachweis eines Rezidivs basiert in der Regel auf einem messbaren Tg-Spiegel mit einer steigenden Tendenz. Diskutiert wird die Relevanz eines Anstiegs der Anti-Tg-Antikörper [94].

Sofern nach Einschätzung des behandelnden Arztes bei niedrig messbaren Tg-Werten (z. B. während der TSH-Suppression ein Tg unter $1 \mathrm{ng} / \mathrm{ml}$ bzw. während der TSH-Stimulation ein $\mathrm{Tg}$ unter $10 \mathrm{ng} / \mathrm{ml}$ ) kein ausreichender Rezidivverdacht für die Durchführung einer erneuten RIT besteht, ist in der Regel eine abwartende Haltung mit kurzfristigen Kontrollen des Tg-Spiegels einer I-131 dxGKS vorzuziehen.

Hinweis: Die Konstellation erhöhter Thyreoglobulin-Spiegel ohne und ggf. auch mit geringem Korrelat in der I-131 rxGKS erfordert zum Ausschluss lod-negativer Metastasen den Einsatz weiterer bildgebender Verfahren (z. B. MRT von Hals und Mediastinum, CT Thorax, Skelettszintigrafie, F-18 FDG-PET/CT oder F-18 FDG PET/MRT).

\section{Kontraindikationen}

\section{dxGKS}

Absolut: Schwangerschaft, Stillzeit. Über einen Zeitraum von etwa 3 Monaten sollte vor einer I-131 dxGKS bzw. I-131-Therapie abgestillt worden sein, um die Strahlenexposition der Mammae zu reduzieren. Die Evidenz für die Angabe eines zeitlichen Intervalls entspricht einem Expertenkonsens [2, 31, 32].

Relativ: eingeschränkte Eignung für die Aufnahme auf einer nuklearmedizinischen Station.

\section{rXGKS}

Siehe Verfahrensanweisung zur Radioiodtherapie beim differenzierten Schilddrüsenkarzinom [15].

\section{Durchführung der Untersuchung}

\section{Patientenvorbereitung}

- Das Einverständnis des Patienten zur Durchführung der Untersuchung/Behandlung soll schriftlich dokumentiert sein. Die Aktivität darf erst nach Aufklärung des Patienten über die spezifischen Risiken der beabsichtigten Strahlenanwendung verabreicht werden.

- Die I-131 dxGKS mit Aktivitäten im Bereich von etwa $150 \mathrm{MBq}$ bis etwa $370 \mathrm{MBq}$ l-131 ist unter stationären Bedingungen auf einer nuklearmedizinischen Therapiestation durchzuführen [80-82]. Bei der Aktivitätsbemessung sind die individuelle Fragestellung sowie die Abbildungseigenschaften der Gammakamera und der SPECT(/CT) zu berücksichtigen.

In Vorbereitung auf eine I-131 dxGKS ist eine exogene oder endogene TSH-Stimulation erforderlich. Die beiden Methoden der TSH-Stimulation vor einer RIT sind in der entsprechenden Verfahrensanweisung zur Radioiodtherapie beschrieben [15]. Bei niedriger Wahrscheinlichkeit für iodavide Metastasen sollte die rhTSH-Stimulation bevorzugt werden. Hingegen ist rhTSH derzeit nicht für die Radioiodtherapie von Organmetastasen zugelassen und der Einsatz von rhTSH zur Therapie bekannter Fernmetastasen stellt einen „off-label-use“ dar. Ein Schilddrüsenhormonentzug ist bei Verdacht auf eine persistierende oder rezidivierte Erkrankung zu erwägen, falls eine Radioiodtherapie in unmittelbarem Anschluss an die dxGKS in Betracht kommt. Zielgröße ist ein TSH-Anstieg auf $30 \mathrm{mU} / \mathrm{I}$ nach dem Schilddrüsenhormon-Entzug mit oder ohne überbrückende Gabe von Triiodthyronin. In der retrospektiven Analyse der Daten aus dem Würzburger und Münsteraner Register wurde bei einer endogenen TSH-Stimulation mit Werten von 20-30 mU/l ein gleich hoher Ablationserfolg erzielt wie bei TSH-Werten über $30 \mathrm{mU} / \mathrm{l}$ [97].

FRAGE 4

Welche TSH-Stimulation sollte bei der I-131 dxGKS bevorzugt werden?

Kommentar: Bei niedriger Wahrscheinlichkeit für iodavide Metastasen sollte die TSH-Stimulation durch rekombinantes humanes TSH bevorzugt werden. Bei höherer Wahrscheinlichkeit für eine Metastasierung sollte der Arzt mit dem Patienten ein individuelles Behandlungskonzept besprechen.

- Nach erhöhter lodexposition sollte auf ein ausreichendes Zeitintervall bis zur Ganzkörperszintigrafie geachtet und ggf. die lodurie bestimmt werden [54, 
61, 76]. lodhaltige Medikamente (z. B. Desinfektionsmittel, Augentropfen, lodid-Medikation) oder Nahrungsmittel (z. B. Multivitamin- und SpurenelementKombinationen, Seetang) sind 4-6 Wochen vor der Untersuchung zu vermeiden. Nach Applikation von Röntgenkontrastmitteln hatte sich nach einem Zeitraum von einem Monat in den Studien von Sohn et al. [76] und Padovani et al. [61] bzw. nach einem Zeitraum von 4 bis 6 Wochen in der Studie von Mishra et al. [54] die lodausscheidung mit dem Urin wieder normalisiert. Da aber iodavides Gewebe nach der Gabe von iodhaltigem Röntgenkontrastmittel noch länger abgesättigt sein kann, sollte ein Mindestabstand zwischen der Gabe iodhaltiger Röntgenkontrastmittel und der l-131 dxGKS von mindestens 2 Monaten gewährt werden. Wesentlich längere Zeitintervalle sind nach Amiodaron (biologische Halbwertszeit ca. ein halbes Jahr) zu beachten. Eine zusätzliche iodarme Diät über 2 Wochen vor einer Radioiodgabe wird empfohlen. Das kurzzeitige Absetzen einer Levothyroxin-Medikation vor rhTSH-Gabe, z. B. beginnend wenige Tage vor der ersten rhTSH-Injektion, ist eine weitere Möglichkeit, die lodzufuhr prätherapeutisch zu verringern [49]. Es existiert aber keine Evidenz, dass durch ein kurzzeitiges Absetzen von Levothyroxin die Sensitivität der dxGKS erhöht wird.

- Bei weiblichen Patienten muss eine Schwangerschaft ausgeschlossen sein. Eine Stillperiode soll - wenn möglich für den Zeitraum von 3 Monaten [2, 31, 32] - vor einer I-131-Applikation beendet worden sein. Hierdurch wird die Strahlenexposition der Mammae begrenzt. In einem kürzeren Abstand nach dem Abstillen ist die lodaufnahme in der Brust noch erhöht, was zu einer entsprechend höheren Strahlenexposition der Mammae führt. Im Falle einer I-131 Applikation in diagnostischer Aktivität ist analog zur Radioiodtherapie bei benignen Schilddrüsenerkrankungen die Kontrazeption für 4 Monate bei Patientinnen und Patienten zu empfehlen.

- Bei den seltenen zerebralen oder spinalen Metastasen mit Kompressionsgefahr kann die prophylaktische Gabe von Glukokortikoiden zum Einsatz kommen. Eine neurochirurgische Interventionsnotwendigkeit ist zu prüfen. Dies gilt sowohl für die l-131 dxGKS als auch für die RIT mit anschließender rxGKS und sowohl in Hypothyreose als auch unter rhTSH. Absolute und relative Kontraindikationen der Glukokortikoidtherapie (z. B. Diabetes mellitus, Ulcus ventriculi, Ulcus duodeni, Elektrolytstörungen) sind zu berücksichtigen.

- Reichliche Mahlzeiten können die Resorption des oral applizierten Radioiods vermindern oder verzögern. Die Patienten sollten daher vier Stunden vor sowie etwa eine Stunde nach der oralen Radioiodapplikation nüchtern bleiben.

- Der überwiegende Teil des verabreichten lodids wird mit dem Urin ausgeschieden. Vor Durchführung der
Ganzkörperszintigrafie ist der Patient aufzufordern, seine Harnblase zu entleeren.

- Ein Teil des lodids wird auch über den Gastrointestinaltrakt ausgeschieden. Deshalb kann ein mildes Abführmittel fakultativ am Tag vor der Ganzkörperszintigrafie verabreicht werden. Dadurch wird die Restaktivität im Darm vermindert und die Bildinterpretation vereinfacht.

- Artefakte durch Kontamination sind durch Wechsel der Kleidung und ggf. Waschen/Duschen vor Durchführung der GKS zu vermeiden.

- Zur Vermeidung unklarer Anreicherungen im Bereich des Ösophagus ist es sinnvoll, den Patienten vor der szintigrafischen Aufnahme trinken zu lassen.

- Der Zeitpunkt der Blutentnahme zur Messung des Thyreoglobulin-Spiegels sollte bei Schilddrüsenhormonkarenz und Hypothyreose so gewählt werden, dass bei deutlich erhöhtem Tg-Wert ggf. im nächsten Schritt eine I-131-Therapie erfolgen kann. Bei exogener Stimulation sollte die Tg-Messung drei Tage nach der zweiten Injektion von rhTSH erfolgen.

- Der Einfluss einer Stimulation des Speichelflusses nach Radioiodapplikation auf die Strahlenexposition der Speicheldrüse und damit die Wahrscheinlichkeit der Induktion einer Speicheldrüsendysfunktion wird derzeit in der Literatur kontrovers diskutiert. Die Reduktion der Strahlenexposition durch eine Beschleunigung der Exkretion könnte durch eine erhöhte Extraktion aus dem Plasma nach Stimulation überkompensiert werden. Eine abschließende Bewertung und Empfehlung ist derzeit nicht möglich.

\section{Notwendige Vorabinformationen}

- Schilddrüsenspezifische Anamnese (Voroperationen, vorangegangene Radioiodtherapien, Recurrensparese, Hypoparathyreoidismus, etc.).

- Falls keine exogene Stimulation durch rhTSH erfolgt: seit wann besteht Schilddrüsenhormonkarenz?

- Zeitpunkt der letzten Exposition mit iodhaltigem Röntgenkontrastmittel oder iodhaltigen Medikamenten; Zeitpunkt des Beginns einer iodarmen Diät.

- Krankheitsspezifische Untersuchung.

- Operationsbericht (Vollständigkeit der Schilddrüsenresektion, Anzahl und Lokalisation der entfernten Lymphknoten, möglichst mit Nennung der Kompartimente). Beim follikulären Schilddrüsenkarzinom Angaben, ob eine Infiltration über die Tumorkapsel hinaus und/oder eine Angioinvasion vorgelegen hatte.

- Histopathologie (Tumorentität, Tumorstadium, Tumordurchmesser, Kapselinfiltration, Anzahl der untersuchten Lymphknoten, Lymphknoteninfiltration, Differenzierungsgrad, Verwendung der TNM-Klassifikation gemäß der 8. Auflage der UICC [75, 89]).

- Laborwerte (TSH-Konzentration, Thyreoglobulin mit Wiederfindung, Anti-Thyreoglobulin-Antikörper, ggf. Messung der lodurie, Kreatinin, Differentialblutbild, 
im Falle eines postoperativen Hypoparathyreoidismus Calcium, anorganisches Phosphat, Parathormon).

- Ultraschallbefund des Halses.

- Befunde vorangegangener I-131 rxGKS oder I-131 dxGKS.

- Ggf. Befunde anderer vorangegangener bildgebender Verfahren. Hinweis: Besteht aufgrund der Risikoeinschätzung, insbesondere bei einem ansteigenden Thyreoglobulin-Spiegel und einer vorangegangenen negativen I-131 Szintigrafie der begründete Verdacht auf nicht-iodspeichernde Metastasen, ist ergänzend eine Positronen-Emissions-Tomographie (PET)/CT oder -/MRT mit F-18-Fluorodeoxyglucose (FDG) indiziert [17, 19, 23, 33, 44, 68]. Potenzieller Vorteil der F-18 FDG-PET/CT bzw. der F-18 FDG PET/MRT ist eine Tracer-Speicherung in der Mehrzahl der iodnegativen Metastasen [18, 39]. Dabei erhöht eine exogene TSHStimulation wahrscheinlich die Sensitivität der Untersuchung. Bei Verdacht auf Lungenmetastasen wird eine CT des Thorax (ohne Kontrastmittel) empfohlen.

- Kürzlich applizierte Radiopharmaka.

- Bei Frauen im gebärfähigen Alter und erhaltener Fertilität: Aktueller Schwangerschaftstest, Laktationsstatus.

\section{FRAGE 5}

Welcher zeitliche Abstand sollte zwischen einer kontrastverstärkten CT und einer I-131 dxGKS gewahrt werden?

Kommentar: Die dxGKS wird nach einer zumindest 8-wöchigen lodkarenz oder später durchgeführt [54, 61, 76]. In diesem Zeitraum kann die morphologische und übrige funktionelle Bildgebung komplettiert werden, so dass dringliche Behandlungsindikationen (z. B. stabilisationsgefährdende Knochenmetastasen, intraspinale Raumforderungen, zerebrale Metastasen) nicht verzögert behandelt werden.

\section{Radiopharmaka}

Wie bei der I-131 rxGKS, bei der das Radiopharmakon durch die Therapie vorgegeben ist, wird auch bei diagnostischer Anwendung üblicherweise lod-131 Nal verabreicht. Bei bestimmten Fragestellungen kann alternativ lod-123 Nal zur Szintigrafie benutzt werden. Ergibt sich z. B. aufgrund eines ansteigenden Thyreoglobulin-Spiegels oder aus Befunden bildgebender Verfahren ein begründeter Verdacht auf eine Metastasierung, besteht die Möglichkeit einer Ganzkörperszintigrafie mit etwa 100 MBq I-123 oder höher [21]. Die prätherapeutische, quantitative Szintigrafie der Halsregion zur Diagnostik des Schilddrüsenrests vor der initialen RIT kann mit 10 bis $40 \mathrm{MBq}$ l-123 erfolgen. Da l-123 nur wenig Teilchenstrahlung emittiert, ist die Strahlenexposition geringer als bei Anwendung von I-131 und eine Beeinträchtigung der lodkinetik durch Vor- schädigung des Zielgewebes ist nicht zu erwarten [34, 50, 74]. Nachteilig gegenüber der I-131-Szintigrafie sind die zeitlich begrenzte Verfügbarkeit von I-123, die höheren Kosten, das aufgrund der physikalischen Halbwertszeit kurze Zeitfenster der Akquisition und die geringere Sensitivität der I-123-Szintigrafie.

Dem diagnostischen Einsatz von I-131 prinzipiell gleichwertig ist die PET/CT mit l-124. Ein relevantes Stunning ist durch Anwendung des Positronen-Emitters aufgrund des im Vergleich zu l-131 kleineren Dosiskoeffizienten und der geringen Aktivität von etwa $25 \mathrm{MBq}$ I-124 nicht zu erwarten [20]. I-124 ist mit einer Halbwertszeit von 4,15 Tagen für dosimetrische Messungen gut geeignet und die PET/CT bzw. PET/MRT weist eine höhere Ortsauflösung und Bildqualität als die Szintigrafie mit I-131 oder I-123 auf. Allerdings sind bisher nur kleine Fallzahlen zum Vergleich von $\mathrm{I}-124$, I-131 und I-123 publiziert worden, wobei die I-124 PET/CT bezüglich der Sensitivität der Szintigrafie mit I-131 oder l-123 in einer kleineren Kohorte überlegen erschien [11]. Wegen der gleichwertigen Sensitivität und der geringeren Exposition im Zielgewebe und im Restkörper kann die I-124 PET/CT in allen genannten Indikationen zur Radioioddiagnostik zum Einsatz kommen. Da allerdings I-124 nach dem Arzneimittelgesetz (AMG) nicht über eine Zulassung verfügt und darüber hinaus nicht allgemein verfügbar ist, wird seine Verwendung auch weiterhin einigen wenigen spezialisierten Zentren als individuelle Behandlungsmaßnahme vorbehalten bleiben.

\section{I-131-Aktivitäten bei diagnostischer Anwendung}

Die Verwendung von Aktivitäten im Bereich von etwa $150 \mathrm{MBq}$ bis etwa $370 \mathrm{MBq}$ l-131 zur dxGKS mit dem Ziel, Restspeicherung im Ganzkörper auszuschließen, erfordert gemäß den Empfehlungen der Strahlenschutzkommission und der Richtlinie Strahlenschutz in der Medizin einen mindestens 48-stündigen stationären Aufenthalt auf einer nuklearmedizinischen Therapiestation mit Abwasser-Abklinganlage [80-82]. Innerhalb dieser 48 Stunden werden mehr als $90 \%$ des verabreichten I- 131 wieder ausgeschieden.

FRAGE 6

Mit welcher Aktivität soll die I-131 dxGKS durchgeführt werden?

Kommentar: Für die dxGKS zum Nachweis oder Ausschluss iodavider Metastasen kommen Aktivitäten von etwa $150 \mathrm{MBq}$ bis etwa $370 \mathrm{MBq}$ l-131 zum Einsatz. Bei der Aktivitätsbemessung sind die individuelle Fragestellung sowie die Abbildungseigenschaften der Gammakamera und der SPECT(/ $\mathrm{CT})$ zu berücksichtigen. 
Wie bei einer RIT ist auch bei der diagnostischen Anwendung die verwendete Aktivität vorab in einem Aktivimeter zu messen und zu dokumentieren. Bei der Verordnung der Aktivität ist das initiale Risikoprofil, der Verlauf der Tg-Werte, der Befund der I-131 rxGKS während der initialen Radioiodtherapie, das Körpergewicht sowie die Abbildungseigenschaften der Gammakamera und der SPECT(/CT) zu berücksichtigen [51, 62, 63].

\section{Strahlenexposition}

Nach oraler Verabreichung wird lodid schnell resorbiert und verteilt sich im austauschbaren Anteil des Körperwassers in einem Verteilungsvolumen, das etwa $40 \%$ der Körpermasse entspricht. Die Ausscheidung erfolgt überwiegend renal. In nicht spezifisch anreichernden Geweben werden die Aktivitätskonzentration und damit die Strahlenexposition durch den jeweiligen Wasseranteil bestimmt. Da der Wasseranteil in fast allen dosisrelevanten Organen um etwa 10-15\% unter dem des Blutes liegt, kann die Blutdosis als geeignetes, jedoch entsprechend überschätzendes Surrogat für die Körperdosis angesehen werden. Gemessene Blutdosen nach Verabreichung von I-131 Nal liegen bei Patienten in der Hypothyreose median im Bereich von etwa 0,09 mGy pro MBq, bei hypothyreosebedingter Beeinträchtigung der Nierenfunktion auch deutlich darüber. Die typische Exposition dosisrelevanter Organe beträgt entsprechend etwa 0,07-0,08 mGy pro MBq, kann aber in anreichernden Geweben (z. B. Gastrointestinaltrakt, Speicheldrüsen, ggf. Mammae) auch höher sein. Die Harnblase ist das höchst exponierte Organ. Da die Miktionsfrequenz einen sehr großen Einfluss auf die Dosis der Blasenwand hat, sollten die Patienten angehalten werden, insbesondere nach einer RIT viel zu trinken und die Blase möglichst oft zu entleeren.

\section{Datenakquisition}

\section{Durchführung der Szintigrafie}

Die lod-131-Szintigrafie erfordert eine Großfeld-Gammakamera mit Hochenergiekollimator. Sofern verfügbar, sollte zur Optimierung der Sensitivität eine Doppelkopfkamera mit „dickem Kristall“ (1/2" entsprechend 1,27 cm oder mehr) verwendet werden. Geeignete Kollimatoren weisen eine geringe Septenpenetration auf; bei Messung mit einer Punktquelle sollte in der resultierenden Verteilung im Szintigramm das Verhältnis der Zehntelwertsbreite (bei 10\% des Maximums) zur Halbwertsbreite etwa zwei betragen, der Faktor drei sollte nicht überschritten werden. Die Halbwertsbreite der Verteilung (Ortsauflösung) bei Messung der Punktquelle in $10 \mathrm{~cm}$ Abstand zum Kollimator beträgt bei einer guten Gammakamera etwa $15 \mathrm{~mm}$ oder besser. Die Aufnahmematrix sollte ausreichend groß sein, um Pixelgrößen von maximal $5 \times 5 \mathrm{~mm}^{2}$ zu erhalten. Während der Akquisition ist das Energiefenster bei 364 keV zentriert. Die Breite des Fensters richtet sich nach der Energieauflösung der Kamera, sie sollte etwa die doppelte Halbwertsbreite der Linie im Energiespektrum betragen. In der Regel sind Fensterbreiten von 15-20\% angemessen.
Bei einer Szintigrafie mit lod-123 sind die Aufnahmeparameter entsprechend anzupassen und es ist ein der Energie angemessener Kollimator, bevorzugt der Mittelenergiekollimator, zu verwenden. Ein Niederenergiekollimator kann verwendet werden, wenn die Septenpenetration nach den oben genannten Kriterien ausreichend gering ist.

Während der Messung sollte der Patient entspannt in Rückenlage bei leichter Reklination des Kopfes liegen.

\section{Zeitpunkt der Ganzkörperszintigrafie}

Die Zeitpunkte der Messungen bei der Ganzkörperszintigrafie richten sich nach der Indikation:

Bei der prätherapeutischen, quantitativen Szintigrafie der Halsregion mit I-131 zur Diagnostik des Schilddrüsenrests vor der initialen RIT ermöglicht eine Messung etwa 24 Stunden nach der Verabreichung die Abschätzung der maximalen Speicherung. Zur Vermeidung eines StunningEffekts ist vor einer RIT die Verwendung von I-123 günstig. Steht hierbei der qualitative Nachweis von lod-positivem Gewebe in der Halsregion im Vordergrund, können die Szintigrafien ab 2 Stunden nach der i.v.-Gabe von I-123 angefertigt werden.

Das Messprogramm für eine Dosimetrie in Vorbereitung auf eine RIT bei iodaviden Metastasen findet sich in der Leitlinie der EANM [45]. Dabei sollte für die Berechnung von Tumordosen eine SPECT/CT Aufnahme [12] mit planaren Aufnahmen kombiniert werden, wobei die Datenakquisition ab spätestens 24 Stunden nach Verabreichung von I-131 beginnt und weitere Szintigramme über die Dauer von mindestens einer effektiven Halbwertszeit angefertigt werden.

Nach einer I-131 Applikation in diagnostischer Dosierung zur visuellen Bewertung des dxGKS kann eine vorgezogene Bildgebung bereits nach 24 Stunden sinnvoll sein, wenn im Falle iodavider Metastasen möglichst früh eine weitere RIT durchgeführt werden soll. Ansonsten sollten die dxGKS 48 bis 72 Stunden nach der I-131 Applikation erfolgen. Dabei ist nach exogener Stimulation mit rhTSH und guter renaler Ausscheidung des lodids eine Messung nach 48 Stunden zu bevorzugen. In Hypothyreose ist bei längeren effektiven Halbwertszeiten für I-131 in Ganzkörper und Zielgewebe die spätere Messung wegen der reduzierten Untergrundaktivität günstiger.

Nach einer RIT oder einer RIT von Metastasen sollten etwa 72 Stunden nach der I-131 Applikation die rxGKS angefertigt werden. Bei guter renaler Clearance unter rhTSH und Therapieaktivitäten um 2 GBq l-131 können die rxGKS bereits nach 48 Stunden akquiriert werden. Bei verzögerter renaler Ausscheidung liefern hingegen spätere Aufnahmen nach 96 Stunden mit niedrigerer Untergrundaktivität bei der rxGKS eine potentiell bessere Erkennbarkeit wenig speicherintensiver Herde. Im Einzelfall kann eine 
rxGKS etwa 1 Woche nach einer therapeutischen lod-131 Applikation zum Nachweis minimal speichernder Metastasen hilfreich sein [35, 47].

Nach der Verabreichung von lod-124 zur PET/CT werden in der Regel Messungen 24 Stunden und 4 Tage nach der Applikation durchgeführt [20, 38].

FRAGE 7

Mit welchem zeitlichen Abstand zur I-131 Applikation soll die dxGKS durchgeführt werden? Kommentar: Die dxGKS wird in der Regel 48 Stunden bis 72 Stunden nach Applikation der I-131-Aktivität angefertigt.

\section{Akquisitionsprotokolle}

Ganzkörperszintigramme (I-131 dxGKS oder I-131 rxGKS) zum visuellen Nachweis oder Ausschluss iodaviden Gewebes sollen in ventraler und dorsaler Sicht den Körper komplett abbilden. Ganzkörperscans sollten mit $\leq 10 \mathrm{~cm}$ pro Minute erstellt werden. Bei planaren Einzelaufnahmen sollte mindestens 10 Minuten gemessen werden. Längere Aufnahmezeiten erhöhen messtechnisch die Sensitivität und sind besonders bei nur noch geringer Restaktivität hilfreich, z. B. bei Szintigrammen, die später als drei Tage nach der Radioiodgabe angefertigt werden. Bei Patienten mit reduzierter renaler Exkretion führt die hohe Aktivitätskonzentration im Gewebe zu einem erhöhten Untergrund und dadurch zu einer reduzierten Sensitivität der Untersuchung. Zur Kompensation kann bei diesen Patienten eine Verlängerung der Aufnahmedauer erwogen werden. Zu vermeiden sind szintigrafische Messungen, welche mit Erreichen einer vordefinierten Impulszahl beendet werden. Hierdurch würde bei hohem Untergrund im Ganzkörper die Aufnahmedauer unnötig verkürzt werden.

Sollte eine Quantifizierung von Speicherherden gewünscht sein, wird neben dem Patienten zur Kalibrierung ein Strahler (I-131, Ba-133) bekannter Aktivität positioniert. Die Aktivität des Strahlers soll ausreichend gering und der Abstand zum Patienten ausreichend groß sein. Nach Möglichkeit sollte zusätzlich zu den planaren Ganzkörperaufnahmen eine SPECT oder eine SPECT/CT des Halses (gوf. unter Einschluss des Thorax) durchgeführt werden, da die Sensitivität der Radioioddiagnostik für den Nachweis von Lymphknotenmetastasen durch die Integration tomographischer Verfahren erhöht werden kann [55].

\section{Interventionen bei unklaren Anreicherungen}

Das Trinken von Flüssigkeit kann helfen, Restaktivität in der Mundhöhle und in der Speiseröhre zu eliminieren. Auf mögliche kutane Kontaminationen ist zu achten; sie können ggf. durch seitliche Aufnahmen oder eine SPECT(/CT) abgeklärt werden.

\section{Befundung und Dokumentation}

1. Visuell Der Befundbericht sollte Angaben zur Lokalisation, Ausdehnung und Intensität der iodspeichernden Herde enthalten. Der qualitative Nachweis einer I-131 Speicherung in Metastasen wird neben der Expression des Natrium-lodid-Symporters durch die Größe des aktiven Tumorgewebes, durch die Höhe der applizierten I-131 Aktivität, durch das Ausmaß und die Dauer der TSH-Stimulation, durch die alimentäre lod-Exposition des Patienten und durch eine zeitlich unmittelbar vorherige diagnostische I-131 Applikation (Stunning) beeinflusst. Ein Vergleich mit früheren dxGKS oder rxGKS ist notwendig. Die Kenntnis des Thyreoglobulin-Spiegels, des Anti-Thyreoglobulin-Antikörperspiegels und des TSH-Spiegels sind für eine Bewertung des szintigrafischen Befundes und die therapeutischen Schlussfolgerungen erforderlich.

2. Quantifizierung Die quantitative Auswertung I-131speichernder Herde in ROI-Technik ist zur Verlaufskontrolle und ggf. zur dosimetrischen Bewertung hilfreich, jedoch nicht obligatorisch. Bei wiederholter dxGKS ist zu beachten, dass die erreichbaren Nachweisgrenzen von der eingesetzten I-131 Aktivität, von der renalen Ausscheidung, von der verwendeten Gammakamera und von der Aufnahmedauer abhängig sein können [12]. Geringe szintigrafische Anreicherungen, die im Verlauf nicht mehr sichtbar sind oder neu auftreten, sind unter Berücksichtigung der jeweils erreichbaren Nachweisgrenzen einer dxGKS zu bewerten.

Restspeicherungen (RIU) im Schilddrüsenrest lassen sich aus der Zahl der im Szintigramm registrierten Nettoereignisse $C_{N}$, der Akquisitionsdauer $t_{a}$, der verabreichten Aktivität A und der Effizienz der Kamera $\varepsilon$ (Ereignisse pro Sekunde/MBq I-131 im Phantom bei 25 mm Schwächungstiefe) berechnen nach RIU $=C_{N} /\left(t_{a} \times A \times \varepsilon\right)$. Die Zahl der Nettoereignisse ergibt sich durch Vergleich der Ereignisse $C_{L}$ in einer ROI der Fläche $F_{L}$ um den Schilddrüsenrest mit den Ereignissen $C_{U}$ in einer ROI der Fläche $F_{U}$ mit repräsentativem Untergrund durch $C_{N}=C-C_{U} \times F_{L} / F_{U}$. Bei fehlender Restspeicherung kann die Nachweisgrenze NG des Szintigramms abgeschätzt werden aus den registrierten Ereignissen C in einer ROI mit etwa $5 \mathrm{~cm}$ Durchmesser über der ehemaligen Schilddrüsenloge nach NG = 5,54/ $\left(t_{a} \times A \times \varepsilon\right) \times \sqrt{C}[13]$.

\section{Inhalte der Bilddokumentation:}

- In digitaler Kopie archiviert und/oder als Papierausdruck und/oder auf

- Röntgenfilm

- Patientendaten zur Identifikation

- Bezeichnung des verabreichten Radiopharmakons

- Applikationsart und verabreichte Aktivität in SI Einheiten (Bq)

- Aufnahmezeitpunkt relativ zur Applikation

- Messdauer in Minuten

- Ggf. ROI-Bild mit Darstellung der verwendeten ROI (Speicherherd und Untergrund) 


\section{Qualitätssicherung}

Zur Qualitätssicherung sind bei den verwendeten Geräten, wie Aktivimeter und Gammakamera, SPECT/CT, regelmäßige Qualitätskontrollen rechtlich vorgeschrieben (Richtlinie Strahlenschutz in der Medizin, Prüfkatalog der Ärztlichen Stellen) [4]. Diese umfassen alle relevanten Parameter wie Untergrundzählrate und Sensitivität, bei Kameras auch die Abbildungseigenschaften. Für Art und Umfang der Prüfungen wird auf die entsprechende Literatur verwiesen, z. B. die DIN 6855-2, eine Empfehlung der Strahlenschutzkommission und die Richtlinie Strahlenschutz in der Medizin [16, 22, 80, 83].

\section{Fehlerquellen}

- Kontamination (u. a. Kleidung, Haut, Haare, Kollimator, Kristall)

- Aktivität im Oesophagus

- Asymmetrische Speicherung in den Speicheldrüsen

- Unspezifische Speicherung u. a. bei pulmonalen Infekten, bei Bronchiektasen, bei Ödemen, in der Mamma, in Nierenzysten und im Thymus

- Bewertung eines zervikalen Herdes als Restschilddrüse oder Lymphknotenmetastase

\section{Offene Fragen}

1. Wirksamkeit des Verzichts auf eine Schilddrüsenhormonmedikation für einige Tage vor einer l-131 Ganzkörperszintigrafie unter rhTSH mit dem Ziel, die lodzufuhr weiter zu verringern.

2. Prognostisch relevante Nachweisgrenzen von Schilddrüsenrestgewebe in der diagnostischen Ganzkörperszintigrafie.

3. Verwendung niedriger I-131 Aktivitäten mit der dezidierten Fragestellung, die erfolgreiche Ablation von Restschilddrüsengewebe zu dokumentieren [30].

4. Integration der Tg-Assays mit sehr hoher Sensitivität in den Behandlungsalgorithmus [25, 26, 28].

\section{Weitere Informationen}

1. Verfahrensanweisung zur Radioiodtherapie beim differenzierten Schilddrüsenkarzinom (Version 4) der Deutschen Gesellschaft für Nuklearmedizin.

2. S2-Leitlinie „Operative Therapie maligner Schilddrüsenerkrankungen, Version vom 09.11.2012“. AWMFRegister Nr. 088/002. Federführende Fachgesellschaft: Deutsche Gesellschaft für Allgemein- und Viszeralchirurgie (DGAV)

3. DGN Handlungsempfehlung (S1-Leitlinie) Radioiodtherapie und diagnostische I-131-Ganzkörperszintigraphi bei differenzierten Schilddrüsenkarzinomen im Kindes- und Jugendalter, Stand 7/2013, AWMF Registernummer: 031-043.

4. EANM-Guidelines for radioiodine therapy of differentiated thyroid cancer [51].
5. EANM dosimetry committee series on standard operational procedures for pre-therapeutic dosimetry I: blood and bone marrow dosimetry in differentiated thyroid cancer therapy [45].

Stand

02. Dezember 2018

Interessenkonflikt

Die Autoren geben an, dass kein Interessenkonflikt besteht.

\section{Korrespondenzadresse}

\section{Prof. Dr. Markus Dietlein}

Klinik und Poliklinik für Nuklearmedizin

Uniklinik Kön

Kerpener Str. 62

50937 Köln

Tel.: 02214785024

E-Mail: markus.dietlein@uk-koeln.de

Literatur

[1] Bachelot A, Leboulleux S, Baudin E et al. Neck recurrence from thyroid carcinoma: serum thyroglobulin and highdose total body scan are not reliable criteria for cure after radioiodine treatment. Clin Endocrinol (Oxf) 2005; 62: 376-379

[2] Bakheet SM, Hammami MM. Patterns of radioiodine uptake by the lactating breast. Eur J Nucl Med 1994; 21: 604-608

[3] Cailleux AF, Baudin E, Travagli JP et al. Is diagnostic iodine-131 scanning useful after total thyroid ablation for differentiated thyroid cancer? J Clin Endocrinol Metab 2000; 85: $175-178$

[4] Campennì A, Giovanella L, Pignata SA et al. Undetectable or low $(<1 \mathrm{ng} / \mathrm{ml})$ postsurgical thyroglobulin values do not rule out metastases in early stage differentiated thyroid cancer patients. Oncotarget 2018; 9(25): 17491-17500

[5] Castro MR, Bergert ER, Goellner JR et al. Immunohistochemical analysis of sodium iodide symporter expression in metastatic differentiated thyroid cancer: correlation with radioiodine uptake. J Clin Endocrinol Metab 2001; 86: 5627-5632

[6] Chen MK, Yasrebi M, Samii ] et al. The utility of I-123 pretherapy scan in $1-131$ radioiodine therapy for thyroid cancer. Thyroid 2012; 22(3): 304-309

[7] Chen L, Luo Q, Shen $Y$ et al. Incremental value of 1311 SPECT/CT in the management of patients with differentiated thyroid carcinoma. J Nucl Med 2008; 49(12): 1952-1957 
[8] Chiovato L, Latrofa F, Braverman LE et al. Disappearance of humoral thyroid autoimmunity after complete removal of thyroid antigens. Ann Intern Med 2003; 139: 346-351

[9] Chung JK, Park Y], Kim TY et al. Clinical significance of elevated level of serum antithyroglobulin antibody in patients with differentiated thyroid cancer after thyroid ablation. Clin Endocrinol (Oxf) 2002; 57: 215-221

[10] de Meer SG, Vriens MR, Zelissen PM et al. The role of routine diagnostic radioiodine whole-body scintigraphy in patients with high-risk differentiated thyroid cancer. J Nucl Med 2011; 52: 56-59

[11] de Pont C, Halders S, Bucerius J et al. (124)I PET/CT in the pretherapeutic staging of differentiated thyroid carcinoma: comparison with posttherapy (131)I SPECT/CT. Eur J Nucl Med Mol Imaging 2013; 40: 693-700

[12] Dewaraja YK, Ljungberg M, Green AJ et al. MIRD pamphlet No. 24: Guidelines for quantitative 1311 SPECT in dosimetry applications. J Nucl Med 2013; 54: 2182-2188

[13] Diess I S, Verburg F, Biko J et al. Improved follow-up of patients with differentiated thyroid carcinoma. The quantitative detection limit of 1311 uptake in diagnostic scans. Nuklearmedizin 2013; 52: 81-87

[14] Dietlein M, Scheidhauer K, Voth E et al. Fluorine-18 fluorodeoxyglucose positron emission tomography and iodine-131 whole-body scintigraphy in the follow-up of differentiated thyroid cancer. Eur J Nucl Med 1997; 24 : 1342-1348

[15] Dietlein M, Eschner W, Grünwald F et al. Procedure guidelines for radioiodine therapy of differentiated thyroid cancer (version 4). [German]. Nuklearmedizin 2016; 55: 77-89

[16] DIN 6855-2 Konstanzprüfung von Einkristall-Gammakameras zur planaren Szintigraphie und zur Einzelphotonen-Emissions-Tomographie mit Hilfe rotierender Messköpfe. 2013. Im Internet: https://dx.doi.org/10.31030/1916487

[17] Feine U, Lietzenmayer R, Hanke JP et al. Fluorine-18-FDG and iodine-131-iodide uptake in thyroid cancer. J Nucl Med 1996;37: 1468-1472

[18] Feine U, Lietzenmayer R, Hanke JP et al. [18FDG whole-body PET in differentiated thyroid carcinoma. Flipflop in uptake patterns of 18FDG and 1311]. Nuklearmedizin 1995;34: 127-134

[19] Freudenberg LS, Frilling A, Kuhl $\mathrm{H}$ et al. Dual-modality FDG-PET/CT in follow-up of patients with recurrent iodinenegative differentiated thyroid cancer. Eur Radiol 2007;17: 3139-3147

[20] Freudenberg LS, Jentzen W, Görges R et al. 124I-PET dosimetry in advanced differentiated thyroid cancer: therapeutic impact. Nuklearmedizin 2007; 46 (4): 121-128

[21] Gerard SK, Cavalieri RR. I-123 diagnostic thyroid tumor whole-body scanning with imaging at 6,24 , and 48 hours. Clin Nucl Med 2002; 27: 1-8

[22] Geworski L, Lottes $G$, Reiners $C$ et al. Empfehlungen zur Qualitätskontrolle in der Nuklearmedizin. Stuttgart: Schattauer, 2009

[23] Giovanella L, Ceriani L, De Palma D et al. Relationship between serum thyroglobulin and 18FDG-PET/CT in 1311-negative differentiated thyroid carcinomas. Head Neck 2012; 34: 626-631

[24] Giovanella L, Suriano S, Ricci R et al. Postsurgical thyroid remnant estimation by $99 \mathrm{mTc}$-pertechnetate scintigraphy predicts radioiodine ablation effectiveness in patients with differentiated thyroid carcinoma. Head Neck 2011; 33 (4): 552-556
[25] Giovanella L, Treglia G, Ceriani L et al. Detectable thyroglobulin with negative imaging in differentiated thyroid cancer patients. What to do with negative anatomical imaging and radioiodine scan? Nuklearmedizin 2014; 53: 1-10

[26] Giovanella L, Treglia G, Sadeghi R et al. Unstimulated highly sensitive thyroglobulin in follow-up of differentiated thyroid cancer patients: a meta-analysis. J Clin Endocrinol Metab 2014; 99: 440-447

[27] Giovanella L, Trimboli P, Verburg FA et al. Thyroglobulin levels and thyroglobulin doubling time independently predict a positive (18)F-FDG PET/CT scan in patients with biochemical recurrence of differentiated thyroid carcinoma. Eur J Nucl Med Mol Imaging 2013; 40: 874-880

[28] Giovanella L, Clark PM, Chiovato L et al. Thyroglobulin measurement using highly sensitive assays in patients with differentiated thyroid cancer: a clinical position paper. Eur J Endocrinol 2014; 171 (2): R33-46

[29] Gotthardt M, Stubinger M, Pansegrau J et al. Decrease of $(99 \mathrm{~m})$ Tc-uptake in autonomous thyroid tissue in Germany since the 1970s. Clinical implications for radioiodine therapy. Nuklearmedizin 2006; 45: 122-125

[30] Haenscheid H, Lassmann M, Buck AK et al. The limit of detection in scintigraphic imaging with I-131 in patients with differentiated thyroid carcinoma. Phys Med Biol 2014; 59: 2353-2368

[31] Hammami MM, Bakheet S. Radioiodine breast uptake in nonbreastfeeding women: clinical and scintigraphic characteristics. J Nucl Med 1996; 37 (1): 26-31

[32] Haugen BR, Alexander EK, Bible KC et al. 2015 American Thyroid Association management guidelines for adult patients with thyroid nodules and differentiated thyroid cancer. Thyroid 2016; 26: 1-133

[33] Helal BO, Merlet P, Toubert ME et al. Clinical impact of (18) F-FDG PET in thyroid carcinoma patients with elevated thyroglobulin levels and negative (131)I scanning results after therapy. J Nucl Med 2001; 42: 1464-1469

[34] Hilditch TE, Dempsey MF, Bolster AA et al. Self-stunning in thyroid ablation: evidence from comparative studies of diagnostic (131)! and (123)I. Eur J Nucl Med Mol Imaging 2002; $29: 783-788$

[35] Hung BT, Huang SH, Huang YE et al. Appropriate time for post-therapeutic I-131 whole body scan. Clin Nucl Med 2009; 34: 339-342

[36] lacobone M, Jansson S, Barczyński M et al. Multifocal papillary thyroid carcinoma - a consensus report of the European Society of Endocrine Surgeons (ESES). Langenbeck's Archives of Surgery 2014; 399 (2): 141-154

[37] Isoda T, Baba S, Maruoka Y et al. Impact of patient age on the iodine/FDG "flip-flop" phenomenon in lung metastasis from thyroid cancer. Ann Nucl Med 2016; 30: 518-24

[38] Jentzen W, Freudenberg L, Eising E et al. Optimized 124I PET dosimetry protocol for radioiodine therapy of differentiated thyroid cancer. J Nucl Med 2008; 49: 1017-1023

[39] Kelders A, Kennes LN, Krohn T et al. Relationship between positive thyroglobulin doubling time and 18F-FDG PET/CTpositive, 1311-negative lesions. Nucl Med Commun 2014; 35: $176-181$

[40] Kim TY, Kim WB, Kim ES et al. Serum thyroglobulin levels at the time of 131I remnant ablation just after thyroidectomy are useful for early prediction of clinical recurrence in lowrisk patients with differentiated thyroid carcinoma. J Clin Endocrinol Metab 2005; 90: 1440-1445

[41] Kim WG, Yoon JH, Kim WB et al. Change of serum antithyroglobulin antibody levels is useful for prediction of 
clinical recurrence in thyroglobulin-negative patients with differentiated thyroid carcinoma. J Clin Endocrinol Metab 2008; 93: 4683-4689

[42] Klubo-Gwiezdzinska J, Van Nostrand D, Atkins F et al. Efficacy of dosimetric versus empiric prescribed activity of 131 l for therapy of differentiated thyroid cancer. J Clin Endocrinol Metab 2011; 96: 3217-3225

[43] Kramer JA, Schmid KW, Dralle $\mathrm{H}$ et al. Primary tumour size is a prognostic parameter in patients suffering from differentiated thyroid carcinoma with extrathyroidal growth: results of the MSDS trial. Eur J Endocrinol 2010; 163: 637-644

[44] Larson SM, Robbins R. Positron emission tomography in thyroid cancer management. Semin Roentgenol 2002; 37: 169-174

[45] Lassmann M, Haenscheid H, Chiesa C et al. EANM dosimetry committee series on standard operational procedures for pre-therapeutic dosimetry I: blood and bone marrow dosimetry in differentiated thyroid cancer therapy. Eur J Nucl Med Mol Imaging 2008; 35: 1405-1412

[46] Lassmann M, Luster M, Haenscheid $\mathrm{H}$ et al. Impact of 131 I diagnostic activities on the biokinetics of thyroid remnants. J Nucl Med 2004; 45: 619-625

[47] Lee JW, Lee SM, Koh GP et al. The comparison of (131)। whole-body scans on the third and tenth day after (131)I therapy in patients with well-differentiated thyroid cancer: preliminary report. Ann Nucl Med 2011; 25: 439-446

[48] Lloyd RV, Osamura RY, Klöppel G et al. WHO Classification of Tumours of Endocrine Organs. Fourth Edition. Volume 10. International Agency for Research on Cancer (IARC), World Health Organization (WHO). 2017; www.iarc.fr

[49] Loeffler M, Weckesser M, Franzius C et al. lodine excretion during stimulation with rhTSH in differentiated thyroid carcinoma. Nuklearmedizin 2003; 42: 240-243

[50] Lundh C, Lindencrona U, Postgard P et al. Radiation-induced thyroid stunning: differential effects of (123)I, (131)I, $(99 \mathrm{~m}) \mathrm{Tc}$, and (211)At on iodide transport and NIS mRNA expression in cultured thyroid cells. J Nucl Med 2009; 50: $1161-1167$

[51] Luster M, Clarke SE, Dietlein M et al. Guidelines for radioiodine therapy of differentiated thyroid cancer. Eur J Nucl Med Mol Imaging 2008; 35: 1941-1959

[52] Mallick U, Harmer C, Yap B et al. Ablation with low-dose radioiodine and thyrotropin alfa in thyroid cancer. $\mathrm{N}$ Engl J Med 2012; 366: 1674-1685

[53] Mazzaferri EL, Robbins RJ, Braverman LE. Authors' response: a consensus report of the role of serum thyroglobulin as a monitoring method for low-risk patients with papillary thyroid carcinoma. J Clin Endocrinol Metab 2003; 88: 4508-4509

[54] Mishra A, Pradhan PK, Gambhir S et al. Preoperative contrast-enhanced computerized tomography should not delay radioiodine ablation in differentiated thyroid carcinoma patients. J Surg Res 2015; 193 (2): 731-737

[55] Mustafa M, Kuwert T, Weber K et al. Regional lymph node involvement in $\mathrm{T} 1$ papillary thyroid carcinoma: a bicentric prospective SPECT/CT study. Eur J Nucl Med Mol Imaging 2010; 37: 1462-1466

[56] Nemec J, Rohling S, Zamrazil V et al. Comparison of the distribution of diagnostic and thyroablative I-131 in the evaluation of differentiated thyroid cancers. J Nucl Med 1979; 20: 92-97

[57] Netzwerk Onkologische Spitzenzentren. Netzwerk-SOP differenziertes Schilddrüsenkarzinom, Diagnose, Therapie und Nachsorge, Version 1.0. Im Internet: www.ccc-netzwerk.de/netzwerk-sops, Stand: 15.10.2018

[58] Pacini F, Capezzone M, Elisei R et al. Diagnostic 131-iodine whole-body scan may be avoided in thyroid cancer patients who have undetectable stimulated serum tg levels after initial treatment. J Clin Endocrinol Metab 2002; 87: 1499-1501

[59] Pacini F, Mariotti S, Formica $\mathrm{N}$ et al. Thyroid autoantibodies in thyroid cancer: incidence and relationship with tumour outcome. Acta Endocrinol (Copenh) 1988; 119: 373-380

[60] Pacini F, Schlumberger M, Dralle $\mathrm{H}$ et al. European consensus for the management of patients with differentiated thyroid carcinoma of the follicular epithelium. Eur J Endocrinol 2006; 154: 787-803

[61] Padovani RP, Kasamatsu TS, Nakabashi CC et al. One month is sufficient for urinary iodine to return to its baseline value after the use of water-soluble iodinated contrast agents in post-thyroidectomy patients requiring radioiodine therapy. Thyroid 2012; 22 (9): 926-930

[62] Perros P, Colley S, Boelart K et al. British Thyroid Association guidelines for the management of thyroid cancer. Clin Endocrinol (Oxf) 2014; 81 (Suppl 1): 1-122

[63] Phan TT, van Tol KM, Links TP et al. Diagnostic I-131 scintigraphy in patients with differentiated thyroid cancer: no additional value of higher scan dose. Ann Nucl Med 2004; 18: 641-646

[64] Pineda JD, Lee T, Ain K et al. lodine-131 therapy for thyroid cancer patients with elevated thyroglobulin and negative diagnostic scan. J Clin Endocrinol Metab 1995; 80 : 1488-1492

[65] Riemann B, Kramer JA, Schmid KW et al. Risk stratification of patients with locally aggressive differentiated thyroid cancer. Results of the MSDS trial. Nuklearmedizin 2010; 49: 79-84

[66] Riemann B, Uhrhan K, Dietlein M et al. Diagnostic value and therapeutic impact of 18F-FDG-PET/CT in differentiated thyroid cancer. Results of a German multicentre study. Nuklearmedizin 2013; 52: 1-6

[67] Robbins RJ, Chon JT, Fleisher $\mathrm{M}$ et al. Is the serum thyroglobulin response to recombinant human thyrotropin sufficient, by itself, to monitor for residual thyroid carcinoma? J Clin Endocrinol Metab 2002; 87: 3242-3247

[68] Robbins R], Wan Q, Grewal RK et al. Real-time prognosis for metastatic thyroid carcinoma based on 2-[18 F]fluoro-2deoxy-D-glucose-positron emission tomography scanning. J Clin Endocrinol Metab 2006; 91: 498-505

[69] Salvatori M, Rufini V, Reale F et al. Radio-guided surgery for lymph node recurrences of differentiated thyroid cancer. World J Surg 2003; 27: 770-775

[70] Schlumberger M, Berg G, Cohen O et al. Follow-up of lowrisk patients with differentiated thyroid carcinoma: a European perspective. Eur J Endocrinol 2004; 150: 105-112

[71] Schlumberger M, Catargi B, Borget I et al. Strategies of radioiodine ablation in patients with low-risk thyroid cancer. N Engl J Med 2012; 366: 1663-1673

[72] Schmidt D, Szikszai A, Linke R et al. Impact of 131। SPECT/ spiral CT on nodal staging of differentiated thyroid carcinoma at the first radioablation. J Nucl Med 2009; 50 (1): $18-23$

[73] Shattuck TM, Westra WH, Ladenson PW et al. Independent clonal origins of distinct tumor foci in multifocal papillary thyroid carcinoma. N Engl J Med 2005; 352 (23): 24062412 
[74] Siddiqi A, Foley RR, Britton KE et al. The role of 123I-diagnostic imaging in the follow-up of patients with differentiated thyroid carcinoma as compared to 1311 -scanning: avoidance of negative therapeutic uptake due to stunning. Clin Endocrinol (Oxf) 2001; 55: 515-521

[75] Sobin LH, Gospodarowicz MK, Wittekind C. TNM classification of malignant tumours. 7th ed. New-York: Wiley-Blackwell, 2009

[76] Sohn SY, Choi JH, Kim NK et al. The impact of iodinated contrast agent administered during preoperative computed tomography scan on body iodine pool in patients with differentiated thyroid cancer preparing for radioactive iodine treatment. Thyroid 2014; $24: 872-877$

[77] Spanu A, Solinas ME, Chessa F et al. 131/ SPECT/CT in the follow-up of differentiated thyroid carcinoma: incremental value versus planar imaging. J Nucl Med 2009; 50 (2): 184-190

[78] Spanu A, Nuvoli S, Gelo I et al. Role of Diagnostic ${ }^{131}$ I SPECT/ CT in Long-Term Follow-up of Patients with Papillary Thyroid Microcarcinoma. J Nucl Med 2018; 59 (10): 1510-1515

[79] Spencer CA. Clinical review: Clinical utility of thyroglobulin antibody (TgAb) measurements for patients with differentiated thyroid cancers (DTC). J Clin Endocrinol Metab 2011; 96: 3615-3627

[80] Strahlenschutz in der Medizin - Richtlinie zur Strahlenschutzverordnung (StrlSchV) vom 26. 05.2011 (GMBI 2011, Nr. 44-47, S. 867), zuletzt geändert durch RdSchr. d. BMUB v. 11.07.2014 (GMBI. 2014, Nr.49, S. 1020). Im Internet: https://www.bmu.de/gesetz/richtlinie-zur-strahlenschutzverordnung

[81] Strahlenschutzgesetz (StrlSchG) - Gesetz zum Schutz vor der schädlichen Wirkung ionisierender Strahlung, zuletzt geändert durch Artikel 2 des Gesetzes vom 27.06.2017 (BGBI.I S. 1966). Im Internet: www.gesetze-im-internet.de/ strlschg; Stand 15.10.2018

[82] Strahlenschutzkommission. Notwendigkeit der stationären Durchführung der Ganzkörperszintigraphie mit I-131 beim Schilddrüsenkarzinom. Empfehlung der Strahlenschutzkommission. Verabschiedet in der 190. Sitzung der SSK am 22.|23. April 2004. Bundesanzeiger 2004; im Internet: https://www.bundesanzeiger.de/ebanzwww/

[83] Strahlenschutzkommission. Qualitätskontrolle von nuklearmedizinischen Geräten - Festlegung von Reaktionsschwellen und Toleranzgrenzen. Verabschiedet in der 243. Sitzung der Strahlschutzkommission am 16./17. September 2010. 2010; im Internet: https://www.bundesanzeiger.de/ ebanzwww/

[84] Sugg SL, Ezzat S, Rosen IB et al. Distinct multiple RET/PTC gene rearrangements in multifocal papillary thyroid neoplasia. J Clin Endocrinol Metab 1998; 83 (11): 4116-4122

[85] Szujo S, Sira L, Bajnok L. The impact of post-radioiodine therapy SPECT/CT on early risk stratification in differentiated thyroid cancer; a bi-institutional study. Oncotarget 2017; 8 (45):7 9825-79834

[86] Thies ED, Tanase K, Maeder U et al. The number of 131 । therapy courses needed to achieve complete remission is an indicator of prognosis in patients with differentiated thyroid carcinoma. Eur J Nucl Med Mol Imaging 2014; 41: 2281-2290

[87] Torlontano M, Crocetti U, D’Aloiso L et al. Serum thyroglobulin and 131 I whole body scan after recombinant human TSH stimulation in the follow-up of low-risk patients with differentiated thyroid cancer. Eur J Endocrinol 2003; 148: $19-24$
[88] Tuttle RM, Tala H, Shah J et al. Estimating risk of recurrence in differentiated thyroid cancer after total thyroidectomy and radioactive iodine remnant ablation: using response to therapy variables to modify the initial risk estimates predicted by the new American Thyroid Association staging system. Thyroid 2010; 20: 1341-1349

[89] Tuttle RM, Haugen B, Perrier ND. Updated American Joint Committee on Cancer/Tumor - Node - Metastasis staging system for differentiated and anaplastic thyroid cancer (eighth edition): what changed and why? Thyroid 2017; 27 (6): 751-756

[90] Van Nostrand D, Khorjekar GR, O’Neil J et al. Recombinant human thyroid-stimulating hormone versus thyroid hormone withdrawal in the identification of metastasis in differentiated thyroid cancer with 131I planar whole-body imaging and 124I PET. J Nucl Med 2012; 53 (3): 359-362

[91] Van Nostrand D. Selected Controversies of Radioiodine Imaging and Therapy in Differentiated Thyroid Cancer. Endocrinol Metab Clin North Am 2017; 46 (3): 783-793

[92] Verburg FA, de Keizer B, Lips C], Zelissen PM, de Klerk JM. Prognostic significance of successful ablation with radioiodine of differentiated thyroid cancer patients. Eur J Endocrinol 2005; 152: 33-37

[93] Verburg FA, Haenscheid H, Biko J et al. Dosimetry-guided high-activity (131)I therapy in patients with advanced differentiated thyroid carcinoma: initial experience. Eur J Nucl Med Mol Imaging 2010; 37: 896-903

[94] Verburg FA, Luster M, Cupini C et al. Implications of thyroglobulin antibody positivity in patients with differentiated thyroid cancer: a clinical position paper. Thyroid 2013; 23 : 1211-1225

[95] Verburg FA, Stokkel MP, Duren C et al. No survival difference after successful (131)I ablation between patients with initially low-risk and high-risk differentiated thyroid cancer. Eur J Nucl Med Mol Imaging 2010; 37: 276-283

[96] Verburg FA, Verkooijen RB, Stokkel MP et al. The success of 1311 ablation in thyroid cancer patients is significantly reduced after a diagnostic activity of $40 \mathrm{MBq} 131 \mathrm{l}$. Nuklearmedizin 2009; 48: 138-142

[97] Vrachimis A, Riemann B, Mäder U et al. Endogenous TSH levels at the time of ${ }^{131}$ I ablation do not influence ablation success, recurrence-free survival or differentiated thyroid cancer-related mortality. Eur J Nucl Med Mol Imaging 2015 [Epub ahead of print] DOI: 10.1007/s00259-015-3223-2

[98] Winter M, Winter J, Heinzel A et al. Timing of post $131 \mathrm{l}$ ablation diagnostic whole body scan in differentiated thyroid cancer patients. Less than four months post ablation may be too early. Nuklearmedizin 2015; 54: 151-157

[99] Yap BK, Murby B. No adverse effect in clinical outcome using low preablation diagnostic 1311 activity in differentiated thyroid cancer: refuting thyroid-stunning effect. J Clin Endocrinol Metab 2014, 99 (7): 2433-2440

[100] Youngwirth LM, Adam MA, Scheri RP et al. Extrathyroidal Extension Is Associated with Compromised Survival in Patients with Thyroid Cancer. Thyroid 2017; 27 (5): 626-631

Bibliografie

DOI https://doi.org/10.1055/a-0891-1839

Online-Publikation: 29.04.2019

Nuklearmedizin 2019; 58: 228-241

(C) Georg Thieme Verlag KG Stuttgart · New York ISSN 0029-5566 